\title{
FIRST ASSESSMENTS ON HERITAGE SCIENCE ORIENTED IMAGE-BASED MODELING USING LOW-COST MODIFIED AND MOBILE CAMERAS
}

\author{
E. Adamopoulos $* 1$, F. Rinaudo ${ }^{2}$, A. Bovero ${ }^{3}$ \\ ${ }^{1}$ Department of Computer Science, Università degli Studi di Torino, Corso Svizzera 185, Torino (TO), Italy \\ ${ }^{2}$ Department of Architecture and Design, Politecnico di Torino, Viale Mattioli 39, Torino (TO), Italy \\ ${ }^{3}$ Fondazione Centro Conservazione e Restauro dei Beni Culturali "La Venaria Reale", Via XX Settembre 18, Venaria (TO), Italy \\ efstathios.adamopoulos@unito.it, fulvio.rinaudo@unito.it, alessandro.bovero@centrorestaurovenaria.it
}

\section{Commission II}

KEY WORDS: Heritage, Modeling, Modified Camera, Mobile Camera, Multi-Spectral, Thermal, Low-Cost, Structure-from-Motion

\begin{abstract}
:
Three-dimensional modeling of cultural heritage, especially concerning large scale studies, as for example, archaeometry, diagnostics and conservation intervention applications, which usually require high-resolution and multi-spectral analyses, necessitates the use of complicate and often expensive equipment. Recent developments regarding low-cost commercially available spectrally modified digital reflex cameras, smartphones with good quality image sensors, mobile thermal cameras in combination with automated or semiautomated photogrammetric software implementing Structure from Motion (SfM) and Multiview Stereo (MVS) algorithms constitute some cheaper and simpler alternatives. Although, the results of the integration of these types of sensors and techniques are often not evaluated as metric products. The presented research combines the above-mentioned instrumentation and software to implement and evaluate low-cost 3D modeling solutions on heritage science-oriented case studies, but also to perform some first assessments on the resulting models' metric properties, quality of texture and usefulness for further scientific investigations.
\end{abstract}

\section{INTRODUCTION}

The necessity of 3D heritage digitization can be attributed to the need for comprehensive documentation, protection, preservation interventions and valorisation. It has been highlighted multiple times in international agreements and directives. Application fields include digital archives, 3D virtual display and restoration, diagnosis, conservation and monitoring (Li et al., 2010). Most cases of large scale modeling and visualization, usually require high-resolution and highly accurate digitization products.

Many heritage sciences fields, as for example archaeometry and diagnostics benefit greatly from three-dimensional modeling utilizing data corresponding to different spectra. The classic photogrammetric modeling approach by visible spectrum (VIS) imagery can provide valuable data to further investigate historic surfaces (Cai, 2017) and to support the overall restoration process (Dellepiane et al. 2017). Direct image-based modeling or posttexturing with non-VIS images can provide 3D records useful for art conservation, since near ultraviolet (UVA) and near infrared (IRA) imaging comprise common non-invasive techniques for the study of wall paintings (Piroddi et al., 2016), artworks (Bendada et al., 2015) and archival documents (Vandermeulen et al., 2018). Long-wavelength infrared (IRC) imaging (thermography) is usually associated with assessment of the state of preservation of historic structures to identify concentrations of moisture, cracks and delaminations (Grinzato, 2012). The last three techniques, which produce non optical data, have also been explored for direct 3D modeling or for texturing heritage digital replicas with various approaches (Adamopoulos et al., 2019). Image-to-model (2D-to-3D) registration with corresponding points, combined Bundle Adjustment (BA), bi-camera systems, registration by photogrammetric tracking on pre-calibrated sensors, cloud-to-cloud and model-to-model (3D-to-3D) registration with precise models produced by active sensors are some of those approaches.

Despite the undoubtable usefulness of 3D modeling products containing VIS and non-VIS information oriented for heritage science, these products often require expensive instrumentation and software and very time-consuming processing. Although, recently, simple to use automated or semi-automated photogrammetric software implementing Structure from Motion (SfM) and Multiview Stereo (MVS) algorithms, which are becoming extremely popular for heritage applications, in combination with low-cost sensors, have made heritage digitization more feasible (Georgopoulos and Stathopoulou, 2017). More specifically, the availability of low-cost digital and modified digital cameras for UVA, IRA and multi-spectral imaging, that are not any more restricted to very low resolutions has made possible the direct modeling from non-VIS imagery (Grifoni et al., 2018; Webb et al., 2018). It should also be mentioned that many contemporary mobile phone cameras can produce imagery of adequate quality for photogrammetric use and have already successfully been implemented in case studies for rapid cultural heritage mapping (Calantropio et al., 2017). Additionally, commercially available small mobile thermal cameras can be found on the market, which, despite low resolutions can produce radiometrically accurate thermal depictions $\left( \pm 3 \sim 5^{\circ} \mathrm{C}\right)$ and should therefore also be considered for heritage modeling applications.

The current study aims to investigate the feasibility of large-scale multi-spectral image-based heritage digitization, implementing workflows that utilize low-cost equipment, free/ low-cost SfM approach-based software for 3D modeling and free software for point cloud manipulation and post-processing. As mentioned, recent bibliography provides several case studies evaluating smart phone and low-cost digital cameras for heritage VIS 3D

\footnotetext{
* Corresponding author
} 
modeling (Rehany et al., 2017; Santagati et al., 2017) but the nonVIS products are seldom evaluated from a metric point of view; as has been performed for more complicated and expensive spectral acquisition/ photogrammetric reconstruction scenarios (Mathys et al., 2019). Therefore, this study also addresses in detail all parameters of the sensors utilized, acquisition and processing workflows implemented, and performs an extensive comparison on the geometric and the radiometric characteristics of the three-dimensional results produced.

Section 2 of this paper focuses on the case studies, the low-cost equipment used, acquisition scenarios and other characteristics of produced datasets. Section 3 concentrates on software, hardware, processing workflows for image-based modeling and results. In section 4 the results are assessed, metric comparisons are made, and further heritage-science oriented evaluations are described. Finally, in section 5 some concluding remarks and directions for future research are demonstrated.

\section{DATASETS}

\subsection{Case Studies}

The study objects of this research included a replica of an Early Cycladic II Spedos-variety marble figurine (approx. $4 \mathrm{~cm} \times 4 \mathrm{~cm}$ x $4 \mathrm{~cm}$ ), a copy of a Roman capital (approx. $45 \mathrm{~cm}$ x $45 \mathrm{~cm} \mathrm{x} 45 \mathrm{~cm}$ ) and part of a façade, at the monumental complex of the Reggia di Venaria in Turin, Italy, located in the former $18^{\text {th }}$ century stables and horse-riding school, designed by Benedetto Alfieri and restored in 2005 for the purpose of hosting "La Venaria Reale" Conservation and Restoration Center (approx. $2.5 \mathrm{~m}$ x 4.6m), herein referred to as $\mathrm{OBJ} 1, \mathrm{OBJ} 2$ and $\mathrm{OBJ} 3$ respectively.

\subsection{Instrumentation}

The instrumentation used in this research (table 1) consisted of: a Canon EOS Rebel SL1 used DSLR camera (230\$) with a fullspectrum acquisition conversion (275\$) by 'Life Pixel Infrared' and three external filters ( $76.5 \$$ each) for VIS, IRA and UVA+ blue imaging; a Canon 1200D DSLR camera (220\$); a Canon EF-S 18-55mm IS II lens (90\$); a Huawei P30 phone (485\$) with a 5.6mm lens camera (Sony IMX650 Exmor RS wide sensor); a FLIR One Pro mobile thermal (IRC) camera for Android smart phones (400\$) with thermal resolution of 160 pixels x 120 pixels (saved at a 1440 pixels x 1080 pixels format), accuracy $\pm 3^{\circ} \mathrm{C}$ and thermal sensitivity $150 \mathrm{mK}$; a MANFROTTO Tripod (67.5\$) for cameras and smart phones. Additionally, a STONEX F6 handheld scanner was used for further metric assessments on OBJ2, with $0.5-4.5 \mathrm{~m}$ effective range, up to $2 \%$ upon scanning distance $3 \mathrm{D}$ point accuracy and $5 \mathrm{mrad}$ resolution.

\begin{tabular}{|c|c|c|c|c|}
\hline Type & $\begin{array}{c}\text { Small } \\
\text { DSLR }\end{array}$ & $\begin{array}{c}\text { Small } \\
\text { DSLR }\end{array}$ & $\begin{array}{c}\text { Smart } \\
\text { Phone }\end{array}$ & $\begin{array}{c}\text { Thermal } \\
\text { camera }\end{array}$ \\
\hline Brand & Canon & Canon & Huawei & FLIR \\
\hline Model & $\begin{array}{c}\text { EOS } \\
1200 \mathrm{D}\end{array}$ & $\begin{array}{c}\text { REBEL } \\
\text { SL1 }\end{array}$ & P30 & $\begin{array}{c}\text { One Pro for } \\
\text { Android }\end{array}$ \\
\hline & & & \\
\hline Sensing range & $\begin{array}{c}380- \\
740 \mathrm{~nm}\end{array}$ & $\begin{array}{c}280 \mathrm{~nm}- \\
1.4 \mu \mathrm{m}\end{array}$ & $\begin{array}{c}380- \\
740 \mathrm{~nm}\end{array}$ & $8-14 \mu \mathrm{m}$ \\
\hline Resolution & $17.9 \mathrm{MP}$ & $17.9 \mathrm{MP}$ & $39.9 \mathrm{MP}$ & $1.6 \mathrm{MP}$ \\
\hline Sensor & $\begin{array}{c}\mathrm{CMOS} \\
\text { APS-C }\end{array}$ & $\begin{array}{c}\text { CMOS } \\
\text { APS-C }\end{array}$ & CMOS & $\begin{array}{c}\text { Uncooled } \\
\text { VOx }\end{array}$ \\
\hline Pixel size & $4.31 \mu \mathrm{m}$ & $4.31 \mu \mathrm{m}$ & $0.93 \mu \mathrm{m}$ & $1.33 \mu \mathrm{m}$ \\
\hline
\end{tabular}

\subsection{Data acquisition}

Due to the specific interest in testing and evaluating techniques that differed from established practices on heritage spectral imaging and modeling, the comparability of results was taken into consideration, for planning the acquisition of datasets for image-based approaches with the low-cost sensors. Meaning, that despite following a standard workflow for capturing the image datasets to be processed with SfM approach photogrammetric software, it was attempted to keep as many capturing conditions constant as possible for all sensors and spectra, for every case study. Thus, during the production of the $3 \mathrm{D}$ meshes, the main parameters that would vary and therefore be compared would be only the different wavelengths captured and different processing software. More specifically, an effort was made to keep internal and external parameters similar amongst low-cost sensors and spectra and constant during data acquisition, furthermore, taking into consideration ground sampling distances (GSDs) - density of data. In all cases custom white balance was applies and no further photographic equipment was used. Especially, thermal images were acquired on short spans of time, on stable climatic conditions maintaining same minimum - maximum temperatures of the visualisation scale, to collect uniform IRC data. During thermal acquisition, optical images of the same scenes were stored as well. Moreover, point cloud acquisition of OBJ2 with the handheld scanner was performed taking into consideration the final density. All photo acquisition conditions are summarized in tables 2-4. For OBJ3 a set of 18 control and check points were measured with a GeoMax Zoom30 3", producing results of 5-6 $\mathrm{mm}$ accuracy at $\mathrm{x}, 2-3 \mathrm{~mm}$ at $\mathrm{y}$ and $4-5 \mathrm{~mm}$ at $\mathrm{z}$ axis.

\begin{tabular}{|c|c|c|c|c|c|c|c|c|}
\hline 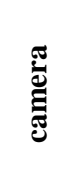 & $\underset{\Xi}{\underline{\Xi}}$ & 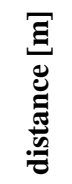 & 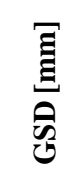 & 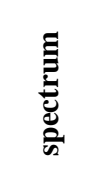 & 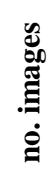 & $\stackrel{0}{0}$ & 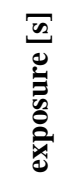 & $\stackrel{\wp}{\Omega}$ \\
\hline SL1 & 18 & 0.20 & 0.048 & VIS & 45 & 8 & $1 / 20$ & 200 \\
\hline SL1 & 18 & 0.20 & 0.048 & full & 45 & 8 & $1 / 20$ & 200 \\
\hline SL1 & 18 & 0.20 & 0.048 & UVAb & 45 & 8 & $1 / 2$ & 200 \\
\hline SL1 & 18 & 0.20 & 0.048 & IRA & 45 & 8 & $1 / 2$ & 200 \\
\hline P30 & 5.6 & 0.25 & 0.044 & VIS & 45 & 8 & $1 / 20$ & 200 \\
\hline
\end{tabular}

Table 2: details of image datasets for OBJ1

\begin{tabular}{|c|c|c|c|c|c|c|c|c|}
\hline 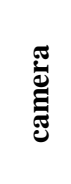 & $\underline{\Xi}$ & 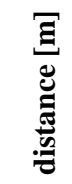 & $\frac{\bar{\Xi}}{\vec{\Xi}}$ & 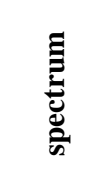 & 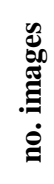 & 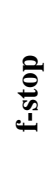 & $\begin{array}{l}\bar{a} \\
0 \\
0 \\
0 \\
0 \\
0\end{array}$ & $\stackrel{\oslash}{\varrho}$ \\
\hline SL1 & 18 & 0.75 & 0.18 & full & 40 & 11 & $1 / 5$ & 200 \\
\hline SL1 & 18 & 0.75 & 0.18 & UVAb & 40 & 11 & 1 & 200 \\
\hline SL1 & 18 & 0.75 & 0.18 & IRA & 40 & 11 & 1 & 200 \\
\hline
\end{tabular}

Table 3: details of image datasets for OBJ2 


\begin{tabular}{|c|c|c|c|c|c|c|c|c|}
\hline 苞 & $\underset{\underline{\Xi}}{\underline{\Xi}}$ & 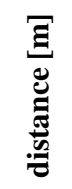 & 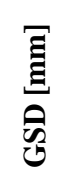 & 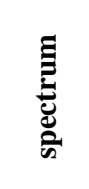 & 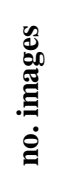 & 兑 & 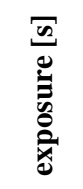 & $\stackrel{\wp}{\varrho}$ \\
\hline $1200 \mathrm{D}$ & 18 & 1.50 & 0.36 & VIS & 55 & 11 & $1 / 15$ & 200 \\
\hline SL1 & 18 & 1.50 & 0.36 & full & 55 & 11 & $1 / 15$ & 200 \\
\hline SL1 & 18 & 1.50 & 0.36 & IRA & 55 & 11 & $1 / 5$ & 200 \\
\hline SL1 & 18 & 1.50 & 0.36 & UV-B & 55 & 11 & $1 / 5$ & 200 \\
\hline P30 & 5.6 & 2.00 & 0.35 & VIS & 55 & 1.8 & $1 / 500$ & 200 \\
\hline FLIR & 1.5 & 1.75 & 1.55 & VIS & 110 & - & - & _ \\
\hline FLIR & - & - & - & IRC & 110 & - & - & - \\
\hline
\end{tabular}

Table 4: details of image datasets for OBJ3

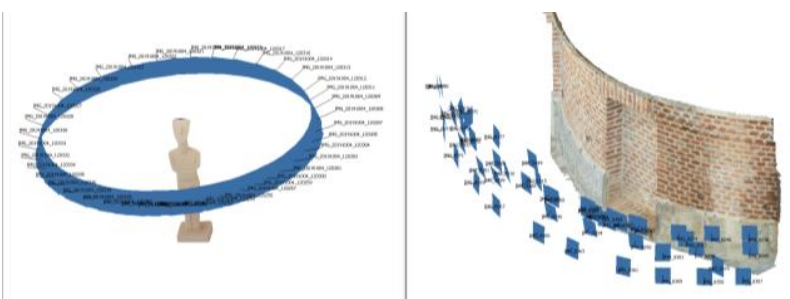

Figure 1: data acquisition scenarios OBJ1(left) and OBJ3(right)

\section{PROCESSING}

\subsection{Software and Hardware}

The software employed for SfM image-based modeling included commercial solutions Agisoft Metashape Standard (179\$) (AM), 3D Flow Zephyr Lite (165\$) (FZ) and a pipeline combining open/ free tools: VisualSfM (VSfM) (Wu, 2013), CMVS (Furukawa et al., 2010) and MeshLab. For the processing of datasets, a SANTECH customized portable laptop was used, with a 6-core Intel i7-8750H CPU at $2.2 \mathrm{GHz}$ (Max $4.1 \mathrm{GHz}$ ) with $32 \mathrm{~GB}$ RAM and NVIDIA GeForce RTX 2070 GPU.

\subsection{Image-Based Modeling Approach}

A standard semi-automatic photogrammetric procedure for largescale heritage documentation was followed for all datasets, maintaining same processing parameters in each software and as similar as possible between software, to be able to compare not only the quality and accuracy of produced clouds and models, but additionally algorithms used, processing times and volumes of produced data. For OBJ1 masking and the highest parameters available were used for clouds and meshes generation. For OBJ2 and $\mathrm{OBJ} 3$ an overview of the processing parameters is presented in the following table (table 5). For the first two objects scaling was performed with use of multiple scales; for OBJ3 referencing was performed using 9 points with known coordinates at a local $\mathrm{x}, \mathrm{y}, \mathrm{z}$ system as control points and 9 for later check. For texture generation a 16,384 x 16,384 resolution JPEG format was chosen in Metashape, Zephyr and MeshLab with mosaicking blend type.

\begin{tabular}{|c|c|c|c|c|}
\hline & \multicolumn{3}{|c|}{ Agisoft Metashape } & 3DFlow Zephyr \\
\hline PARAMETERS & OBJ2 & OBJ3 & OBJ2 & OBJ3 \\
\hline $\begin{array}{c}\text { Matching \& } \\
\text { Alignment }\end{array}$ & \multicolumn{4}{|l|}{} \\
\hline Key point density & high & high & high & high \\
\hline Pair preselection & generic & unordered & generic & unordered \\
\hline Key point limits & $50 \mathrm{~K}$ & $50 \mathrm{~K}$ & $50 \mathrm{~K}$ & $50 \mathrm{~K}$ \\
\hline Tie points limits & $50 \mathrm{~K}$ & accurate & $50 \mathrm{~K}$ & accurate \\
\hline Dense Matching & \multicolumn{5}{|l}{} \\
\hline Masking & no & no & no & no \\
\hline Point density & high & high & medium & medium \\
\hline Depth filtering & moderate & moderate & moderate & moderate \\
\hline Mesh Generation & \multicolumn{5}{|l}{} \\
\hline Max faces number & $10 \mathrm{M}$ & $10 \mathrm{M}$ & $10 \mathrm{M}$ & $10 \mathrm{M}$ \\
\hline Interpolation & disabled & disabled & disabled & disabled \\
\hline
\end{tabular}

\subsection{Processing Results}

\begin{tabular}{|c|c|c|c|c|c|}
\hline & P30 & $\begin{array}{c}\text { SLI } \\
\text { VIS }\end{array}$ & $\begin{array}{c}\text { SL1 } \\
\text { full }\end{array}$ & $\begin{array}{c}\text { SL1 } \\
\text { UVAb }\end{array}$ & $\begin{array}{c}\text { SL1 } \\
\text { IRA }\end{array}$ \\
\hline Tie points & 20,080 & 35,825 & 38,571 & 10,462 & 21,460 \\
\hline Projections & 56,841 & 110,060 & 115,167 & 28,862 & 55,590 \\
\hline $\begin{array}{c}\text { Repr. error } \\
\text { [pixels] }\end{array}$ & 0.454 & 0.300 & 0.328 & 0.704 & 0.822 \\
\hline GSD [mm/pix] & 0.037 & 0.054 & 0.058 & 0.055 & 0.061 \\
\hline $\begin{array}{c}\text { Dense cloud } \\
\text { points [1,000] }\end{array}$ & 2,782 & 1,281 & 1,165 & 1,288 & 1,170 \\
\hline $\begin{array}{c}\text { Mesh faces } \\
{[1,000]}\end{array}$ & 5,000 & 3,133 & 2,849 & 3,114 & 2,797 \\
\hline Mesh quality & high & high & high & medium & medium \\
\hline $\begin{array}{c}\text { Texture quality } \\
\text { hery }\end{array}$ & $\begin{array}{c}\text { very } \\
\text { high }\end{array}$ & $\begin{array}{c}\text { very } \\
\text { high }\end{array}$ & medium & medium \\
\hline $\begin{array}{c}\text { Total time } \\
\text { [mm:ss] }\end{array}$ & $16: 56$ & $15: 37$ & $15: 45$ & $12: 42$ & $08: 01$ \\
\hline $\begin{array}{c}\text { Check scales' } \\
\text { error [mm] }\end{array}$ & 0.171 & 0.122 & 0.155 & 0.110 & 0.207 \\
\hline
\end{tabular}

Table 6: Agisoft Metashape results OBJ1

\begin{tabular}{|c|c|c|c|c|c|}
\hline & P30 & $\begin{array}{c}\text { SLI } \\
\text { VIS }\end{array}$ & $\begin{array}{c}\text { SL1 } \\
\text { full }\end{array}$ & $\begin{array}{c}\text { SL1 } \\
\text { UVAb }\end{array}$ & $\begin{array}{c}\text { SL1 } \\
\text { IRA }\end{array}$ \\
\hline Tie points & 50,335 & 16,492 & 17,525 & 15,866 & 13,365 \\
\hline Projections & 228,832 & 76,052 & 80,684 & 74,034 & 60,798 \\
\hline $\begin{array}{c}\text { Repr. error } \\
\text { [pixels] }\end{array}$ & 0.639 & 0.441 & 0.463 & 0.680 & 0.688 \\
\hline GSD [mm/pix] & 0.042 & 0.057 & 0.057 & 0.057 & 0.057 \\
\hline $\begin{array}{c}\text { Dense cloud } \\
\text { points [1,000] }\end{array}$ & 750 & 287 & 311 & 351 & 347 \\
\hline $\begin{array}{c}\text { Mesh faces } \\
{[1,000]}\end{array}$ & 1491 & 566 & 620 & 697 & 685 \\
\hline $\begin{array}{c}\text { Mesh quality } \\
\text { high }\end{array}$ & $\begin{array}{c}\text { very } \\
\text { high }\end{array}$ & $\begin{array}{c}\text { very } \\
\text { high }\end{array}$ & low & 1 low \\
\hline $\begin{array}{c}\text { Texture quality } \\
\text { high }\end{array}$ & $\begin{array}{c}\text { very } \\
\text { high }\end{array}$ & $\begin{array}{c}\text { very } \\
\text { high }\end{array}$ & medium & medium \\
\hline $\begin{array}{c}\text { Total time } \\
\text { [mm:ss] }\end{array}$ & $75: 16$ & $24: 33$ & $26: 31$ & $24: 43$ & $24: 01$ \\
\hline $\begin{array}{c}\text { Check scales' } \\
\text { error [mm] }\end{array}$ & 0.171 & 0.197 & 0.178 & 0.158 & 0.166 \\
\hline
\end{tabular}

Table 7: 3DFlow Zephyr results OBJ1 
For OBJ1, VSfM was not able to reconstruct the scene for any spectra, but AM and FZ were able to orient all images (tables 6,7 ). AM produced denser sparse clouds by SL1 VIS and SL1 full spectrum results and significantly sparser for other datasets. However, the density of produced dense cloud and 3D mesh for P30 datasets was almost double in volume than spectral datasets from SLI. Total processing times were similar. FZ produced very dense tie points, dense cloud and mesh results for P30 dataset comparing to SL1 ones, which in fact were very similar between them while comparing volumes. Subsequently, also processing time for P30 dataset was three times comparing the amount of time needed for SL1 spectral datasets. In all scenarios for OBJ1 the RMSE for scaled bars used for check was $<0.25 \mathrm{~mm}$. UVAb and IRA point clouds contained a low amount of noise.

\begin{tabular}{|c|c|c|c|}
\hline & SL1 full & SL1 UVAb & SL1 IRA \\
\hline Images Aligned & $40 / 40$ & $40 / 40$ & $40 / 40$ \\
\hline Tie points [1,000] & 45 & 15 & 16 \\
\hline Projections [1,000] & 111 & 32 & 36 \\
\hline Repr. error [pixels] & 0.703 & 1.510 & 1.910 \\
\hline GSD [mm/pix] & 0.162 & 0.174 & 0.193 \\
\hline Dense cloud points [1,000] & 11,197 & 8,385 & 9,515 \\
\hline Mesh faces [1,000] & 9,913 & 9,804 & 10,000 \\
\hline Mesh quality & high & medium & low \\
\hline Texture quality & very high & high & high \\
\hline Total time [mm:ss] & $28: 56$ & $12: 05$ & $11: 45$ \\
\hline
\end{tabular}

Table 8: Agisoft Metashape results OBJ2

\begin{tabular}{|c|c|c|c|c|c|c|}
\hline & \multicolumn{3}{|c|}{ FZ } & \multicolumn{3}{c|}{ VSfM+CMVS } \\
\cline { 2 - 7 } & $\begin{array}{c}\text { SL1 } \\
\text { full }\end{array}$ & $\begin{array}{c}\text { SL1 } \\
\text { UVAb }\end{array}$ & $\begin{array}{c}\text { SL1 } \\
\text { IRA }\end{array}$ & $\begin{array}{c}\text { SL1 } \\
\text { full }\end{array}$ & $\begin{array}{c}\text { SL1 } \\
\text { UVAb }\end{array}$ & $\begin{array}{c}\text { SL1 } \\
\text { IRA }\end{array}$ \\
\hline Images Aligned & $40 / 40$ & $40 / 40$ & $40 / 40$ & $40 / 40$ & $40 / 40$ & $40 / 40$ \\
\hline $\begin{array}{c}\text { Matching time } \\
\text { [mm:ss] }\end{array}$ & $16: 21$ & $08: 22$ & $06: 21$ & $01: 38$ & $00: 58$ & $00: 56$ \\
\hline $\begin{array}{c}\text { Alignment time } \\
\text { [mm:ss] }\end{array}$ & $00: 14$ & $00: 12$ & $02: 45$ & $00: 38$ & $01: 11$ & $00: 44$ \\
\hline Tie points [1,000] & 15 & 9 & 9 & 27 & 13 & 13 \\
\hline Projections [1,000] & 64 & 32 & 32 & 94 & 36 & 36 \\
\hline Repr. error [pixels] & 0.958 & 1.083 & 1.228 & & & \\
\hline $\begin{array}{c}\text { GSD [mm/pix] } \\
\text { Tming }\end{array}$ & 0.161 & 0.170 & 1.850 & & & \\
\hline $\begin{array}{c}\text { Dense cloud time } \\
\text { [mm:ss] }\end{array}$ & $32: 22$ & $20: 22$ & $22: 03$ & $10: 24$ & $07: 59$ & $06: 08$ \\
\hline $\begin{array}{c}\text { Dense cloud points } \\
{[1,000]}\end{array}$ & 1,869 & 740 & 477 & 1,575 & 927 & 480 \\
\hline
\end{tabular}

Table 9: 3DFlow Zephyr and VSfM+CMVS results OBJ2

For OBJ2, ZA and VSfM were able to only partially reconstruct the scene (figure 2) and produced relatively sparse results. For all processing scenarios for $\mathrm{OBJ} 2$ full spectrum sparse clouds had three times the density comparing to those produced by UVAb and IRA images, but dense clouds were of similar densities.

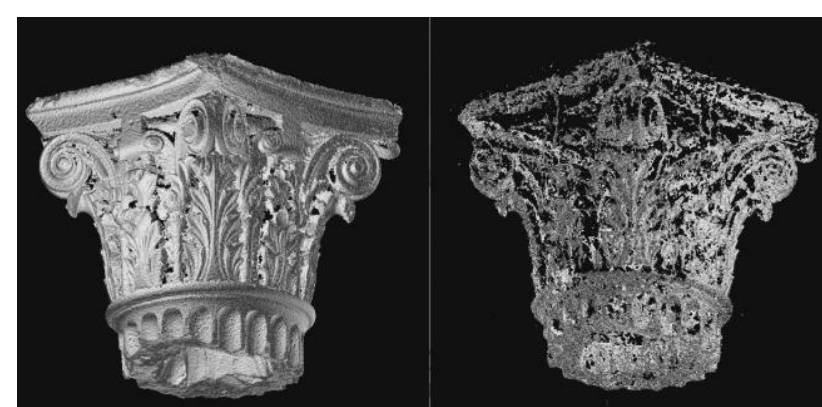

Figure 2: Partially reconstructed scenes of full spectrum (left) and UVAb (right) datasets of OBJ2 with 3DFlow Zephyr

\begin{tabular}{|c|c|c|c|c|c|c|}
\hline & $1200 \mathrm{D}$ & $\begin{array}{l}\text { SL1 } \\
\text { full }\end{array}$ & \begin{tabular}{|l|} 
SL1 \\
IRA \\
\end{tabular} & \begin{tabular}{|c|} 
SL1 \\
UVAb \\
\end{tabular} & P30 & $\begin{array}{l}\text { FLIR } \\
\text { VIS }\end{array}$ \\
\hline \multicolumn{7}{|c|}{ Sparse Cloud } \\
\hline $\begin{array}{l}\text { Images } \\
\text { Aligned }\end{array}$ & $55 / 55$ & $55 / 55$ & $55 / 55$ & $55 / 55$ & $55 / 55$ & $110 / 110$ \\
\hline $\begin{array}{c}\text { Matching time } \\
{[\mathrm{mm}: \mathrm{ss}]}\end{array}$ & $00: 45$ & $00: 48$ & $00: 38$ & $00: 44$ & $01: 44$ & 00:09 \\
\hline $\begin{array}{c}\text { Alignment } \\
\text { time [mm:ss] }\end{array}$ & $00: 40$ & $00: 34$ & $01: 35$ & $00: 33$ & $00: 58$ & $01: 25$ \\
\hline $\begin{array}{c}\text { Tie points } \\
{[1,000]}\end{array}$ & 259 & 275 & 254 & 248 & 211 & 64 \\
\hline $\begin{array}{c}\text { Projections } \\
{[1,000]}\end{array}$ & 942 & 849 & 920 & 880 & 659 & 251 \\
\hline $\begin{array}{l}\text { Repr. error } \\
\text { [pixels] }\end{array}$ & 0.504 & 0.526 & 0.372 & 0.458 & 0.676 & 0.610 \\
\hline $\begin{array}{l}\text { Ground-res } \\
{[\mathrm{mm} / \mathrm{pix}]}\end{array}$ & 0.374 & 0.356 & 0.376 & 0.391 & 0.303 & 1.560 \\
\hline $\begin{array}{c}\text { Average } \\
\text { distance }[\mathrm{m}]\end{array}$ & 1.56 & 1.67 & 1.69 & 1.73 & 1.77 & 1.93 \\
\hline \multicolumn{7}{|c|}{ Dense Cloud } \\
\hline Time $[\mathrm{mm}: \mathrm{ss}]$ & $06: 53$ & $04: 21$ & $05: 29$ & 05:03 & 13:08 & $02: 58$ \\
\hline $\begin{array}{l}\text { Point count } \\
(1,000)\end{array}$ & 6,257 & 8,135 & 7,273 & 6,709 & 11,555 & 482 \\
\hline \multicolumn{7}{|c|}{ Mesh } \\
\hline Time $[\mathrm{mm}: \mathrm{ss}]$ & 01:55 & 03:05 & 02:06 & 01:54 & 03:41 & 00:03 \\
\hline $\begin{array}{c}\text { Face count } \\
(1,000)\end{array}$ & 9,976 & 9,998 & 9,981 & 9,996 & 9,763 & 1,167 \\
\hline Quality & \begin{tabular}{|l|} 
very \\
high \\
\end{tabular} & $\begin{array}{l}\text { very } \\
\text { high } \\
\end{array}$ & $\begin{array}{l}\text { very } \\
\text { high } \\
\end{array}$ & \begin{tabular}{|l|} 
very \\
high \\
\end{tabular} & high & medium \\
\hline \multicolumn{7}{|c|}{ Texture } \\
\hline Time $[\mathrm{mm}: \mathrm{ss}]$ & 09:06 & 08:15 & 08:34 & 09:11 & $20: 37$ & $01: 27$ \\
\hline Quality & $\begin{array}{l}\text { very } \\
\text { high } \\
\end{array}$ & high & high & high & high & low \\
\hline $\begin{array}{l}\text { Total time } \\
\text { [mm:ss] }\end{array}$ & $19: 19$ & $17: 03$ & $18: 22$ & $17: 25$ & $40: 08$ & 06:02 \\
\hline \begin{tabular}{|c|} 
Control points \\
RMSE [mm] \\
\end{tabular} & 1.306 & 1.693 & 1.602 & 1.749 & 3.836 & 3.645 \\
\hline \begin{tabular}{|l|} 
Check points \\
RMSE [mm]
\end{tabular} & 1.089 & 1.954 & 1.434 & 1.619 & 2.663 & 3.133 \\
\hline
\end{tabular}

Table 10: Agisoft Metashape results OBJ3

For OBJ3, AM was able to reconstruct entirely the scene with similar results for the reprojection errors, the density of tie points and densified clouds and the time needed for each processing step of the photogrammetric procedure of all datasets, barring for total time of P30 dataset processing. Control and check points RMSE for $3 \mathrm{D}$ coordinates remained below $2 \mathrm{~mm}$ for all spectral imagery captured by the two DSLRs and bellow $4 \mathrm{~mm}$ for both the data 
captured by the Huawei P30 camera and the visible sensor of the FLIR One Pro thermal camera. All those errors were lower that the accuracy of performed topographic measurements. Although, the imagery from the latter sensor was included in the photogrammetric procedure only to be able to use the computed orientations to texture with thermal data the high-quality 3D mesh produced by the EOS 1200D VIS image dataset. This was performed without optically calibrating the IRC sensor, considering that any distortions would be insignificant on the approximately $5 \mathrm{~cm}$ GDS thermal images. However, the optical sensor of FLIR One Pro was calibrated with the Single Camera Calibrator App of MATLAB.

\begin{tabular}{|c|c|c|c|c|c|}
\hline & 1200D & $\begin{array}{l}\text { SL1 } \\
\text { full }\end{array}$ & $\begin{array}{l}\text { SL1 } \\
\text { IRA }\end{array}$ & \begin{tabular}{|c|} 
SL1 \\
UVAb \\
\end{tabular} & P30 \\
\hline \multicolumn{6}{|c|}{ Sparse Cloud } \\
\hline $\begin{array}{l}\text { Images } \\
\text { Aligned }\end{array}$ & $55 / 55$ & $55 / 55$ & $55 / 55$ & $55 / 55$ & $55 / 55$ \\
\hline \begin{tabular}{|c|} 
Matching time \\
{$[\mathrm{mm}: \mathrm{ss}]$}
\end{tabular} & $23: 19$ & $12: 56$ & $15: 43$ & $12: 56$ & $19: 22$ \\
\hline \begin{tabular}{|c|} 
Alignment \\
time [mm:ss] \\
\end{tabular} & $00: 53$ & $00: 29$ & $00: 22$ & 00:22 & $00: 23$ \\
\hline $\begin{array}{c}\text { Tie points } \\
{[1,000]}\end{array}$ & 33 & 37 & 33 & 36 & 46,614 \\
\hline $\begin{array}{c}\text { Projections } \\
{[1,000]}\end{array}$ & 208 & 147 & 177 & 186 & 277 \\
\hline $\begin{array}{l}\text { Repr. error } \\
\text { [pixels] }\end{array}$ & 0.852 & 0.827 & 0.873 & 1.039 & 1.761 \\
\hline $\begin{array}{l}\text { Ground-res } \\
{[\mathrm{mm} / \mathrm{pix}]}\end{array}$ & 0.428 & 0.396 & 0.412 & 0.488 & 0.324 \\
\hline $\begin{array}{c}\text { Average } \\
\text { distance }[\mathrm{m}]\end{array}$ & 1.687 & 1.654 & 1.721 & 1.738 & 1.964 \\
\hline \multicolumn{6}{|c|}{ Dense Cloud } \\
\hline Time [mm:ss] & $40: 03$ & $46: 08$ & $41: 17$ & $40: 55$ & 107:00 \\
\hline $\begin{array}{l}\text { Point count } \\
(1,000)\end{array}$ & 2,963 & 2,924 & 2,934 & 2,353 & 2,550 \\
\hline \multicolumn{6}{|c|}{ Mesh } \\
\hline Time $[\mathrm{mm}: \mathrm{ss}]$ & 00:29 & $00: 33$ & $00: 30$ & 00:29 & 00:30 \\
\hline $\begin{array}{c}\text { Face count } \\
(1,000)\end{array}$ & 5,897 & 5,795 & 5,450 & 4,646 & 5,025 \\
\hline Quality & high & high & high & high & medium \\
\hline \multicolumn{6}{|c|}{ Texture } \\
\hline Time $[\mathrm{mm}: \mathrm{ss}]$ & $06: 13$ & $07: 49$ & $06: 39$ & $05: 39$ & $10: 44$ \\
\hline Quality & high & high & high & high & medium \\
\hline $\begin{array}{l}\text { Total time } \\
{[\mathrm{mm}: \mathrm{ss}]}\end{array}$ & $70: 57$ & $67: 55$ & $64: 31$ & $60: 21$ & $2: 17: 59$ \\
\hline $\begin{array}{c}\text { Control points } \\
\text { RMSE [mm] }\end{array}$ & 2.068 & 1.551 & 2.131 & 1.606 & 1.160 \\
\hline $\begin{array}{l}\text { Check points } \\
\text { RMSE [mm] }\end{array}$ & 1.940 & 1.668 & 2.318 & 1.665 & 1.279 \\
\hline
\end{tabular}

Table 11: 3DFlow Zephyr results OBJ3

FZ was also able to fully reconstruct OBJ3 producing similar volumes for point clouds and meshes for the datasets from all sensors and spectra. However, tie point count together with reprojection errors were higher for the Huawei P30 imagery and additionally, double the processing time was needed for the full $3 \mathrm{D}$ reconstruction from that dataset. In all cases xyz RMSEs for OBJ3 with FZ were smaller than $2.5 \mathrm{~mm}$.

As presented in images 3-5, VSfM was able to reconstruct only parts of $\mathrm{OBJ} 3$, nevertheless in high detail.

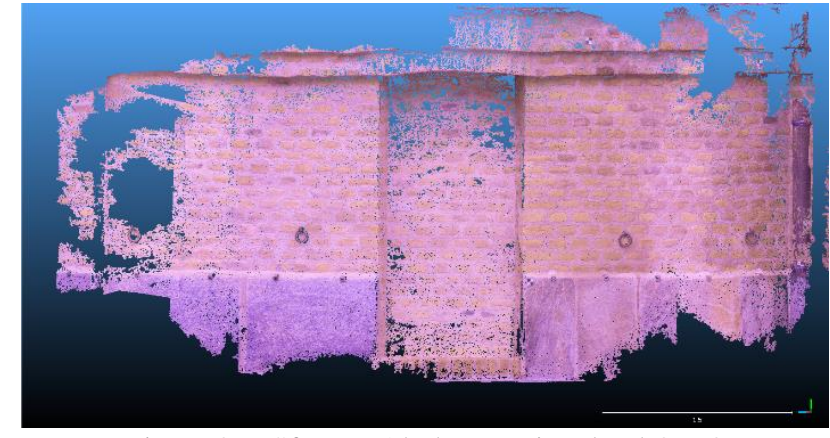

Figure 3: VSfM UVAb dense point cloud OBJ3

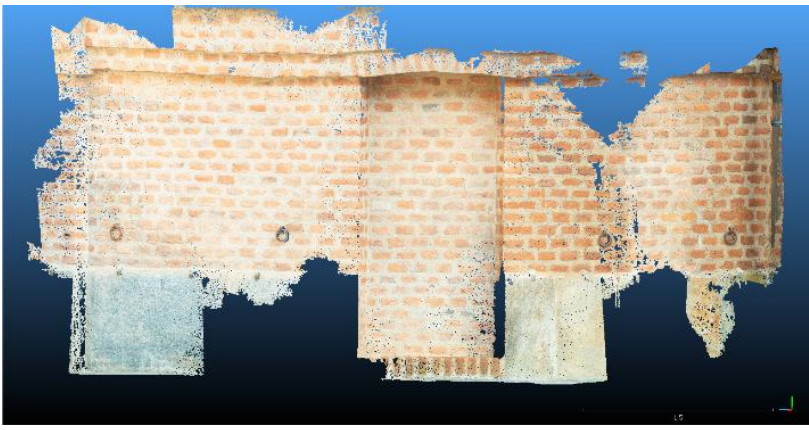

Figure 4: VSfM full spectrum dense point cloud OBJ3

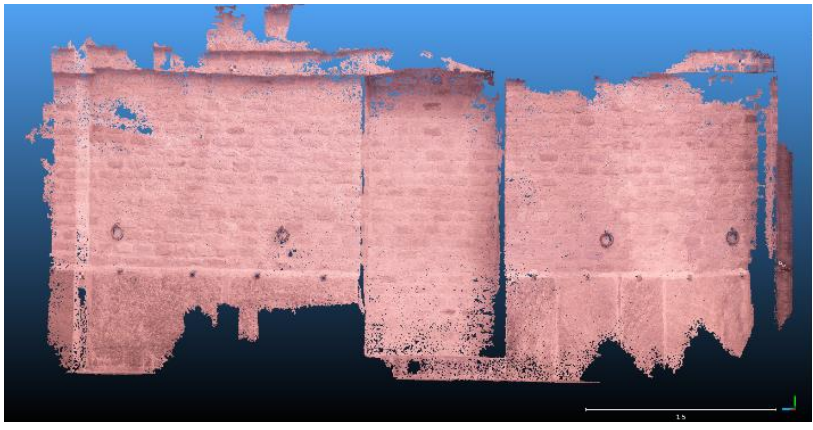

Figure 5: VSfM IRA dense point cloud OBJ3

\section{EVALUATION}

The evaluations conducted regarded quality of produced meshes and textures; considered completeness, preservation of surface details, level of noise, roughness, visual fidelity and radiometric sharpness. Further geometric accuracy tests were performed by comparisons, with SL1 VIS results for OBJ1, with SF6 and veryhigh-resolution photogrammetric results (produced by SfM image-based approach from a Canon 5DS-R 51 MP dense VIS imagery, decimated for this use) for OBJ2 and with very-highresolution photogrammetric results (produced by SfM imagebased approach from a Nikon D800E 36 MP dense VIS imagery, decimated for this use for OBJ3. The comparisons were performed in CloudCompare, by computing Hausdorff distances of $10 \%$ sampled meshes to the reference dense clouds (after ICP Fine registration between them for unreferenced scenes).

For OBJ1, both commercial image-based solutions constructed high-to-very high quality meshes and textures for VIS imagery from SL1 and P30 sensors as well as full spectrum data; for $\mathrm{UVAb}$ and IRA imagery the reconstructed geometries contained a low amount of noise. The significant geometric differences computed between SLI VIS mesh and others can be attributed to the inability of the software to precisely reconstruct the shape of an object with almost flat featureless and colorless surface. 


\begin{tabular}{|c|c|c|c|c|}
\hline SL1 VIS & P30 & SL1 full & SL1 UVAb & SL1 IRA \\
\hline AM & $0.72 / 0.84$ & $0.10 / 0.41$ & $1.12 / 1.37$ & $1.07 / 1.49$ \\
\hline FZ & $0.44 / 1.46$ & $0.90 / 0.40$ & $0.23 / 1.40$ & $0.24 / 1.50$ \\
\hline
\end{tabular}

Table 12: comparisons between SL1 VIS dense cloud and spectral models for OBJ1(mean/RMS distances in $\mathrm{mm}$ )

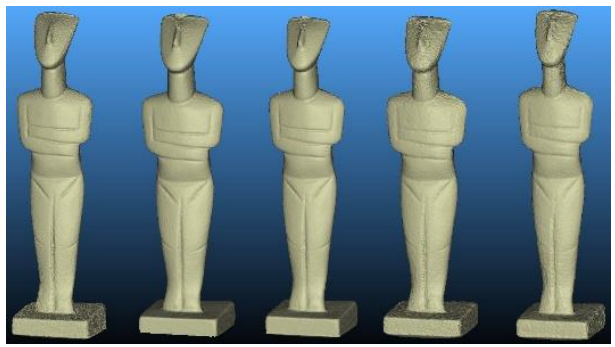

Figure 6: Agisoft Metashape OBJ1 meshes (from left to right) P30, SL1 VIS, SL1 full, SL1 UVAb, SL1 IRA

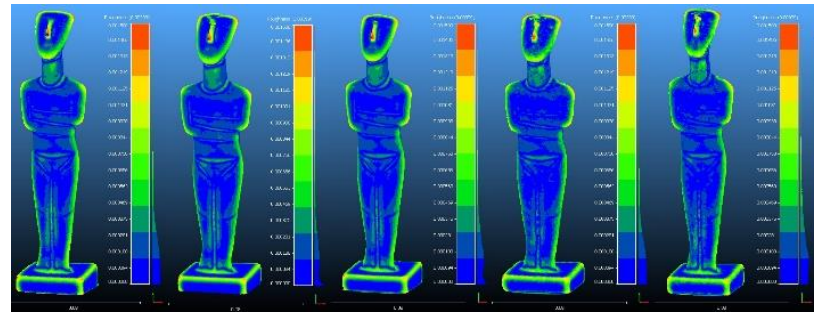

Figure 7: Agisoft Metashape OBJ1 meshes roughness index maps (from left to right) P30, SL1 VIS, SL1 full, SL1 UVAb,

SL1 IRA (calculated for neighbourhoods of $0.5 \mathrm{~cm}^{2}$ )

For OBJ2, AM produced very high-quality $3 \mathrm{D}$ mesh and texture results from full spectrum imagery, medium quality results from UVAb imagery and low-quality results from IRA imagery. For UVAb and IRA imagery the reconstructed geometries contained a very high amount of noise. The inability to autofocus on the object, for a significant part of the data acquisition for these scenarios, under interior lighting conditions certainly contributed to this amount of noise. All mean differences were $<0.5 \mathrm{~mm}$ and RMS $<3 \mathrm{~mm}$, with also $90 \%$ differences $<3 \mathrm{~mm}$ in all cases, which equals to $6 \%$ max error regarding the objects' dimensions.

\begin{tabular}{|c|c|c|c|}
\hline & SL1 & SL1 UVAb & SL1 IRA \\
\hline Canon REBEL-SL1 & - & $0.172 / 1.972$ & $0.656 / 2.580$ \\
\hline Canon EOS5DS R & $-0.387 / 1.941$ & $-0.068 / 2.368$ & $0.523 / 2.817$ \\
\hline SONEX F6 & $-0.095 / 1.923$ & $0.010 / 2.467$ & $0.565 / 2.942$ \\
\hline
\end{tabular}

Table 13: geometric comparisons for OBJ 2 (mean/RMS distances in $\mathrm{mm}$ )

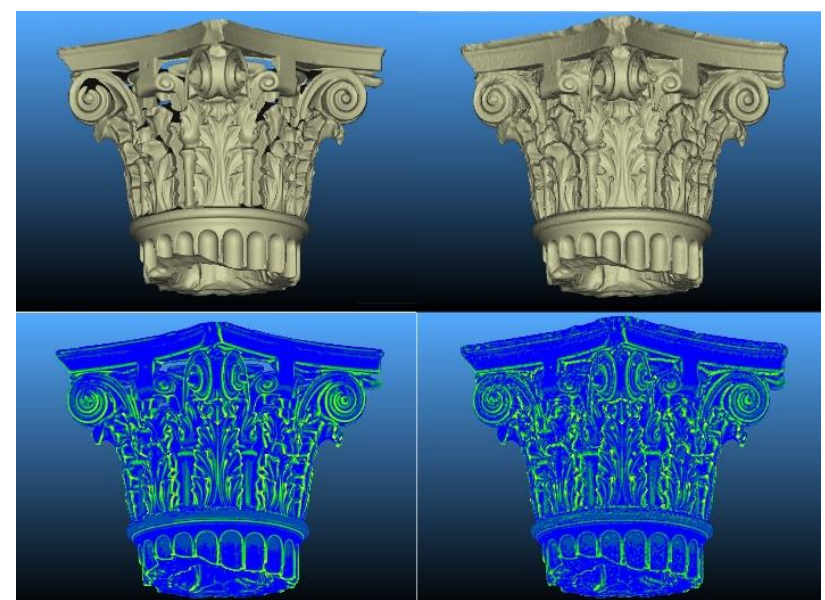

Figure 8: reference models for OBJ 2, meshes (up), roughness index maps (down), SF6 (left), 5DS R VIS (right)

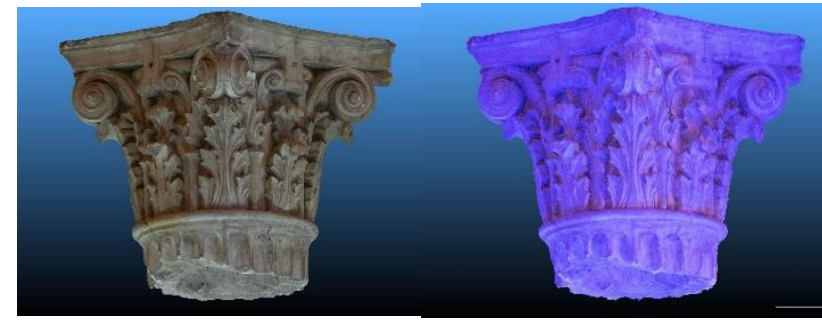

textured mesh SL1 full

textured mesh SL1 UVAb

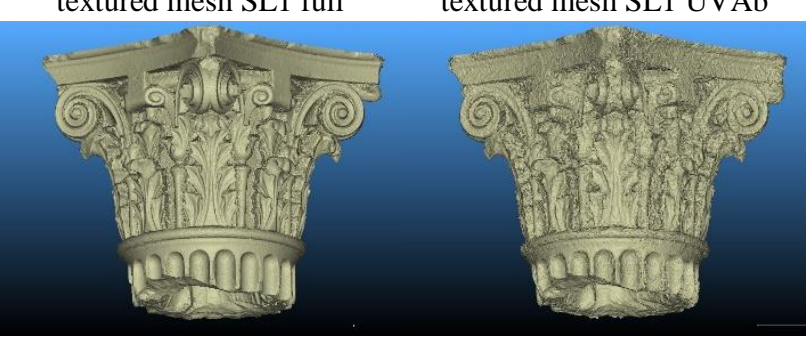

untextured mesh SL1 full

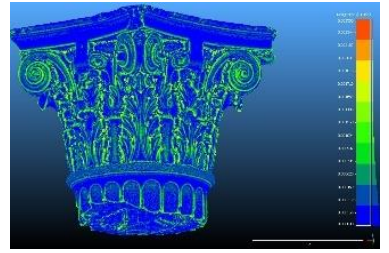

untextured mesh SL1 UVAb
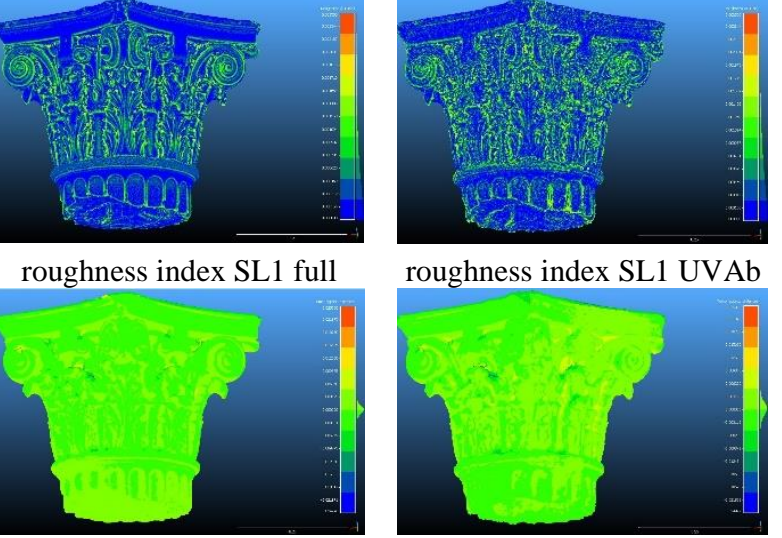

roughness index SL1 UVAb

Hausdorff distances SL1 full Hausdorff distances SL1 UVAb spectrum mesh from SF6 cloud mesh from SF6 cloud

Figure 9: Agisoft Metashape OBJ2 results for full spectrum imagery (left) and UVAb imagery (right)

For OBJ3, both AM and FZ produced high-to-very high-quality 3D meshes and texturing results for all sensors and spectra. Furthermore, P30 imagery produced a very low amount of noise, but for all comparisons with the SL1 VIS data, mean distances were $<1 \mathrm{~mm}$ for both software and RMS < $4 \mathrm{~mm}$ for AMP, $<6$ $\mathrm{mm}$ for FZ, which correspond to approximately $2 \%$ of the smaller dimension of the reconstructed object and, additionally, are lower than the accuracy of the ground control points used for referencing the scene.

\begin{tabular}{|c|c|c|c|c|}
\hline 1200D & P30 & SL1 full & SL1 UVAb & SL1 IRA \\
\hline AM & $-0.572 / 3.589$ & $0.284 / 2.206$ & $-0.946 / 2.053$ & $0.116 / 2.190$ \\
\hline FZ & $-0.020 / 3.882$ & $-0.284 / 5.128$ & $0.307 / 4.129$ & $0.085 / 4.323$ \\
\hline
\end{tabular}

Table 14: geometric comparisons for OBJ3 (mean/RMS distances in $\mathrm{mm}$ )

Meshes produced from DSLR and mobile phone camera image datasets had similar levels of roughness, high preservation of surface details on the final mesh and sharp textures. Moreover, on UVAb and IRA models of the façade, areas of high moisture content, delamination and biodecay are easily distinguishable. The high accuracy 3D model with the low-res thermal texture produced can also assist a more abstract identification of the areas with high surface humidity. 
The International Archives of the Photogrammetry, Remote Sensing and Spatial Information Sciences, Volume XLII-2/W17, 2019 6th International Workshop LowCost 3D - Sensors, Algorithms, Applications, 2-3 December 2019, Strasbourg, France

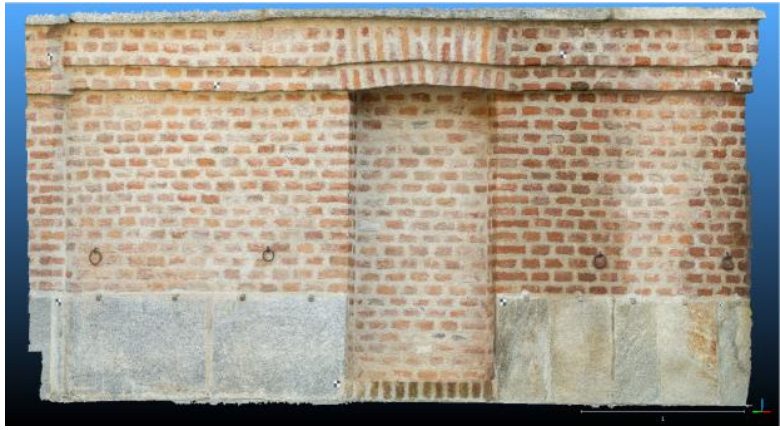

1200D textured mesh

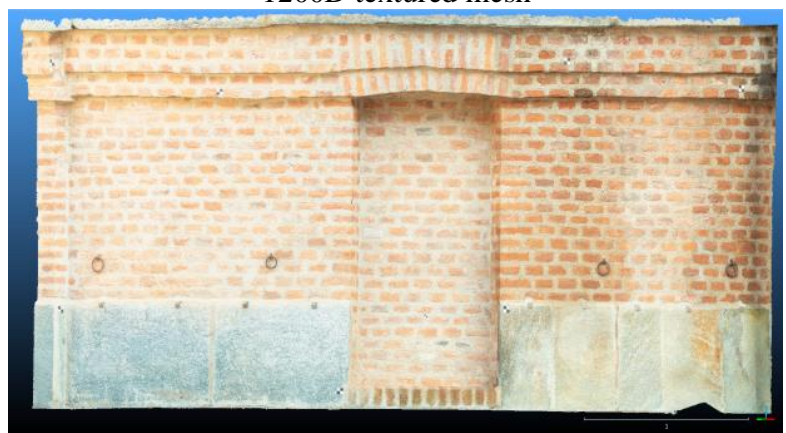

SL1 full spectrum textured mesh

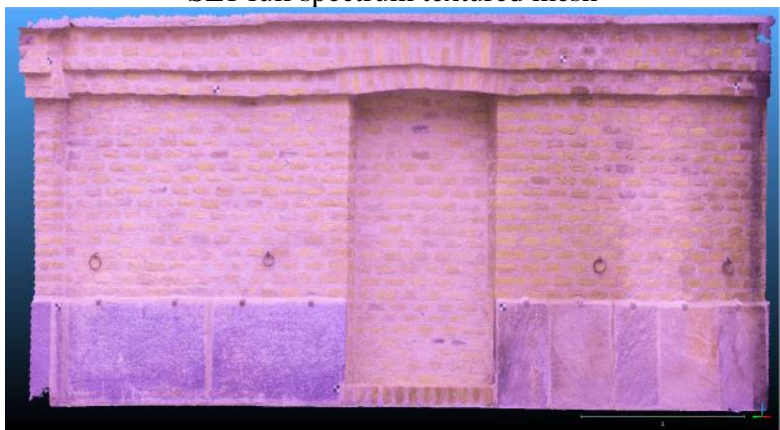

UVAb textured mesh

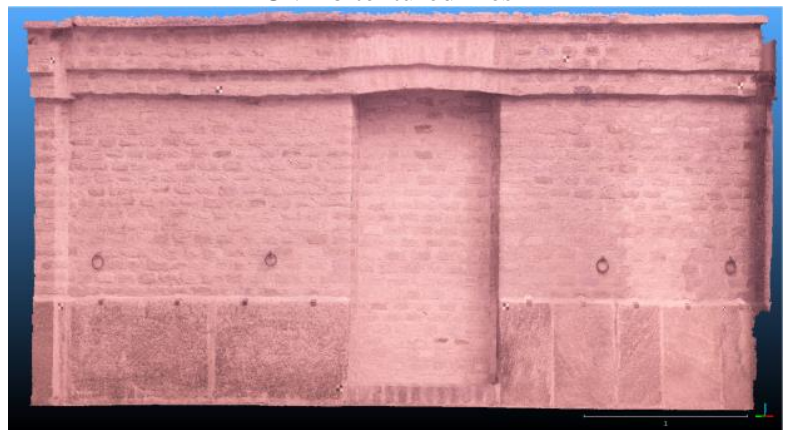

IRA textured mesh

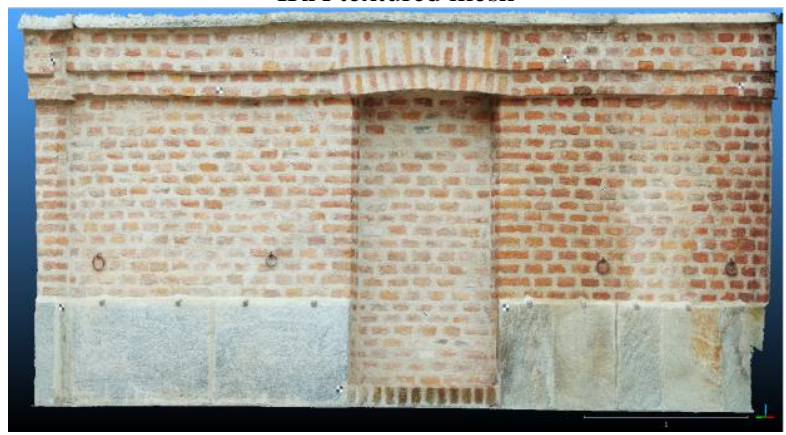

P30 texured mesh

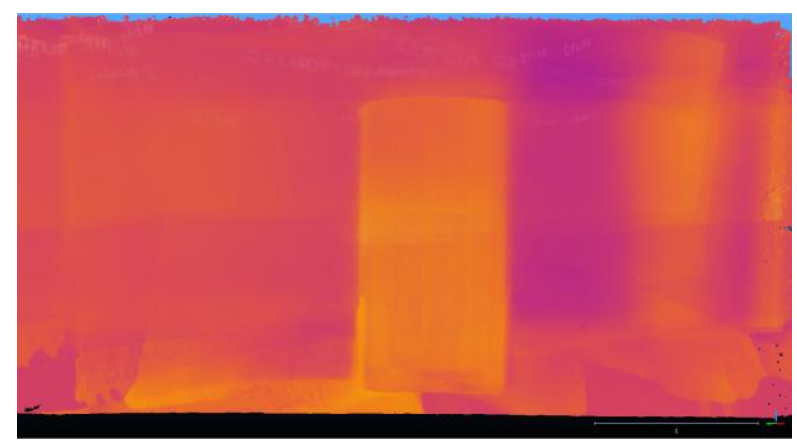

1200D mesh textured with FLIR One Pro IRC imagery

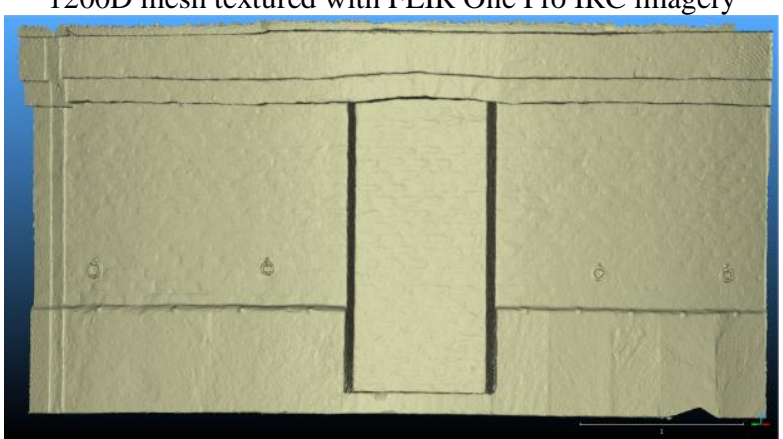

SL1 full spectrum untextured mesh

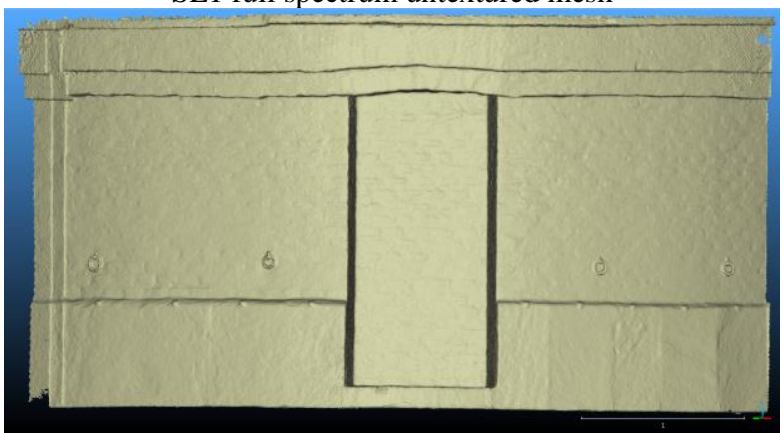

UVAb untextured mesh

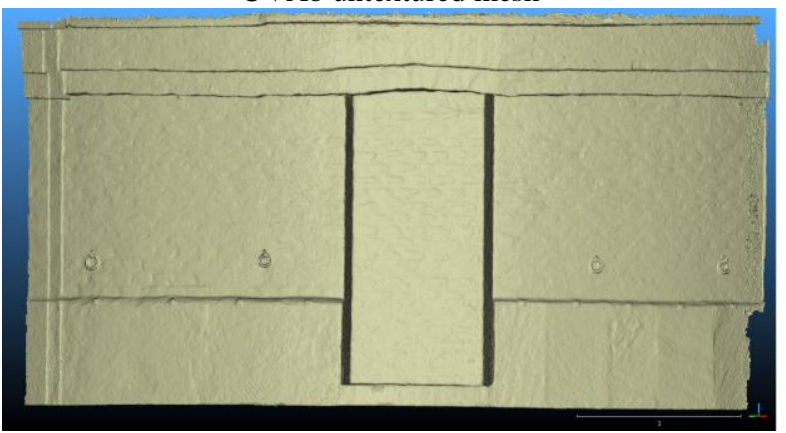

IRA untextured mesh

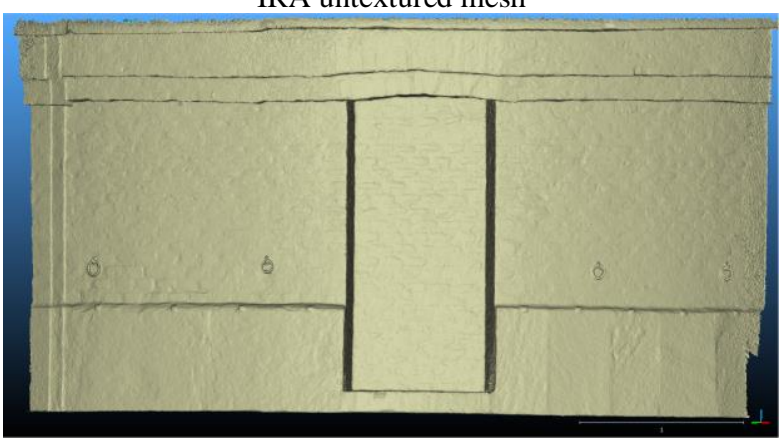

P30 untextured mesh

Figure 8: Agisoft Metashape results for OBJ3 


\section{CONCLUSIONS AND PERSPECTIVES}

The study presented here, aimed to evaluate the use of low-cost photographic and mobile equipment with SfM approach imagebased modeling software, for multi-spectral heritage studies and the first results produced, indeed proved the feasibility of that combination. As anticipated, conditions of data acquisition and characteristics of the case studies, have even greater effect on the photogrammetric pipelines implemented and on the 3D results produced, than when applying standard optical photogrammetric approaches, for large-scale heritage applications. Especially, sharpness, radiometric uniformity and overlaps of the photos are some of the parameters that impacted the quality of spectral meshes produced. The internal alterations of a low-cost DSLR, when modified to be able to capture multi-spectral imagery, mainly regarding focusing, are significant and will further be investigated by the authors along with the impacts of different external filters, lenses and denser image capturing. Even though Metashape was able to fully reconstruct all datasets for case studies involved, other software implemented did not succeed to do so (despite the use of similar algorithms by Zephyr), therefore they should also be further evaluated for low-cost VIS and nonVIS imagery of tangible heritage with denser datasets, other reallife heritage applications and, mainly, on cases where there are significant textures because of deterioration or pigments or deterioration by-products, which have very adverse responses on various spectra. In conclusion, the use of the low-cost equipment implemented here proves promising, considering the very highquality meshes and textures produced by cell phone camera, the ability to distinguish colorings, delaminations, biodecay and moisture from the DSLR non-VIS meshes and moisture content from the model produced with thermal textures (from FLIR One).

\section{ACKNOWLEDGEMENTS}

This project has received funding from the European Union's Framework Programme for Research and Innovation Horizon 2020 (2014 - 2020) under the Marie-Skłodowska Curie Grant Agreement No. $\mathbf{7 5 4 5 1 1 .}$

\section{REFERENCES}

Adamopoulos, E., Rinaudo, F., 2019. 3D Interpretation and Fusion of Multidisciplinary Data for Heritage Science: A Review. Int. Arch. Photogramm. Remote Sens. Spatial Inf. Sci., XLII-2/W15, 17-24, https://doi.org/10.5194/isprs-archivesXLII-2-W15-17-2019, 2019.

Bendada, A., Sfarra, S., Ibarra, C., Akhloufi, M., Pradere, C., Maldague, X., 2015. Subsurface Imaging for Panel Paintings Inspection: A Comparative Study of the Ultraviolet, the Visible, the Infrared and the Terahertz Tpectra. Opto-Electronics Review, 23(1), 90-101, https://doi.org/10.1515/oere-2015-0013.

Cai, Y., 2017. Pattern Discovery from Eroded Rock Art. Digital Imaging for Cultural Heritage Preservation: Analysis, restoration and reconstruction of ancient artworks, 429-447. CRC Press.

Calantropio, A., Colucci, E., Teppati Losè, L., 2017. Rapid Mapping for Built Heritage at Risk using Low-Cost and COTS Sensors. A test in the Duomo Vecchio of San Severino Marche. Int. Arch. Photogramm. Remote Sens. Spatial Inf. Sci., XLII2/W8, 59-66, https://doi.org/10.5194/isprs-archives-XLII-2-W859-2017, 2017.

Dellepiane, M., Callieri, M., Corsini, M., Scopigno R., 2017. Using Digital 3D Models for Study and Restoration of Cultural
Heritage Artifacts. Digital Imaging for Cultural Heritage Preservation: Analysis, restoration and reconstruction of ancient artworks, 39-67. CRC Press.

Furukawa, Y., Curless, B., Seitz, S.M. Szeliski, R., 2010. Towards Internet-Scale Multi-View Stereo. Proceedings of 2010 IEEE Computer Society Conference on Computer Vision and Pattern Recognition, 1434-1441. IEEE.

Georgopoulos, A., Stathopoulou, E.K., 2017. Data Acquisition for 3D Geometric Recording: State of the Art and Recent Innovations. In Heritage and Archaeology in the Digital Age. Acquisition, Curation, and Dissemination of Spatial Cultural Heritage Data, 1-26, https://doi.org/10.1007/978-3-319-653709_1. Springer, Cham.

Grifoni, E., Legnaioli, S., Lorenzetti, G., Pagnotta, S., Palleschi, V., 2017. Image Based Recording of Three-Dimensional Profiles of Paint Layers at Different Wavelengths. Eur J Sci Theol, 13(2), 127-134.

Li, R., Luo, T. Zha, H., 2010. 3D Digitization and Its Applications in Cultural Heritage. EuroMed 2010: Digital Heritage. LNCS, 6436, 381-388. Springer, Cham.

Mathys, A., Semal, P., Brecko, J., Van den Spiegel, D. 2019. Improving 3D Photogrammetry Models through Spectral Imaging: Tooth Enamel as a Case Study. PLoS ONE, 14(8), e0220949, https://doi.org/10.1371/journal.pone.0220949.

Piroddi, L., Ranieri, G., Cogoni, M., Trogu, A., Loddo, F., 2016. Time and Spectral Multiresolution Remote Sensing for the Study of Ancient Wall Drawings at San Salvatore Hypogeum, Italy. Proceedings of Near Surface Geoscience 2016-22nd European Meeting of Environmental and Engineering Geophysics.

Rehany, N., Barsi, A., Tovas, T., 2017. Capturing Fine Details Involving Low-Cost Sensors - A comparative Study. Int. Arch. Photogramm. Remote Sens. Spatial Inf. Sci., XLII-2/W8, 213220, https://doi.org/10.5194/isprs-archives-XLII-2-W8-2132017.

Santagati, C., Lo Turco, M., Bocconcino, M. M., Donato, V., Galizia, M., 2017. 3D Models for All.: Low-Cost Acquisition Through Mobile Devices in Comparison with Image Based Techniques. Potentialities and Weaknesses in Cultural Heritage Domain. Int. Arch. Photogramm. Remote Sens. Spatial Inf. Sci., XLII-2/W8, 221-228, https://doi.org/10.5194/isprs-archivesXLII-2-W8-221-2017.

Vandermeulen, B., Hameeuw, H., Watteeuw, L., Van Gool, L., Proesmans, M., 2018. Bridging Multi-light \& Multi-Spectral Images to Study, Preserve and Disseminate Archival Documents. Proceedings of Archiving 2018 Conference, 64-69, https://doi.org/10.2352/issn.2168-3204.2018.1.0.15. Society for Imaging Science and Technology.

Webb, E.K., Robson, S., MacDonald, L., Garside, D. Evans, R., 2018. Spectral and 3D Cultural Heritage Documentation using a Modified Camera. Int. Arch. Photogramm. Remote Sens. Spatial Inf. Sci., XLII-2, 1183-1190, https://doi.org/10.5194/isprsarchives-XLII-2-1183-2018.

Wu, C., 2013. Towards Linear-Time Incremental Structure from Motion. Proceedings of 2013 Int. Conference on 3D Vision-3DV 2013,127-134. IEEE. 